\title{
TT virus infection: a novel virus-host relationship
}

Virus infections may be short-lived or persistent, subclinical or debilitating or fatal. Virus replication may directly destroy cells, lead to immunologically mediated damage, or be entirely non-cytopathic. The maxim that virus-host relationships evolve from parasitic to commensal, and from commensal to symbiotic over periods of co-habitation, supports the current idealisation of well adapted, successful viruses as being non-harmful, persistent, highly transmissible and frequent in a population. Infections with the eight or more herpesviruses in man each epitomise a successful virus-host relationship, and are the likely outcome of the long period over which these viruses have co-evolved during mammalian evolution [1]. Their narrow species specificity undoubtedly results in part from the sophistication they display in controlling or evading the intracellular defences and the host's immune system, a key component of the virus-host adaptive process. Indeed, the fatal outcome of infection with the macaque virus, cercopithecine herpesvirus 1, after cross-species transmission to man [2] demonstrates how precise the process of host adaptation needs to be. The contrast between the outcomes of infection by several species of simian immunodeficiency virus (SIV) with their natural hosts, such as SIV $_{\mathrm{SM}}$ in sooty mangabeys and SIV $_{\mathrm{CPZ}}$ in chimpanzees, with the AIDS-related illnesses in unnatural hosts (HIV-1 and -2 infection in man, SIV infection in macaques), provides further examples of the influence of adaptation on disease outcome [3].

By any of these general criteria, the recently discovered TT virus (TTV) and relatives appear to have established a remarkably successful interaction with their primate hosts. This short review concentrates on many of the often unique aspects of TTV infections, and hopefully illuminates the ways in which the relationship of TTV infection with human disease may be investigated in the future.

\section{Virology}

TTV is a non-enveloped small virus with a diameter of c. $30-32 \mathrm{~nm}$, and a density of $1.31-1.34 \mathrm{~g} / \mathrm{cm}^{3}$. The TTV genome is $3750-3900$ bases in length and comprises a circular, single-stranded antisense DNA molecule, encoding 4-5 gene sequences [4] (Fig. 1). The largest open reading frame (ORF1) encodes a protein of 770 amino acids with an amino terminus containing multiple positively charged amino acid residues (mainly arginine). This protein is likely to form the nucleocapsid, although details of the expression and function of other proteins are unclear at present.

In overall genome organisation and physical properties, TTV is similar only to a virus infecting poultry, chicken anaemia virus (CAV), currently classified as a member of the circoviridae. This virus family contains a diverse range of small DNA viruses, including porcine circovirus, viruses of many other bird species, and distant relatives in plants (geminiviruses). Without wishing to labour the taxonomy issue, the lumping together of such diverse viruses into one virus family is unhelpful; a more rational classification that reflects both the genome organisation and physical properties of the viruses would split TTV and CAV into one family, and the other animal and bird circoviruses into another.

\section{Genetic diversity}

The most remarkable features of TTV are its prevalence of infection and genetic diversity. Following the development of detection methods that can detect a wide range of different TTV genotypes, it has become apparent that TTV infection is ubiquitous in man, as well as in all other primate species [5]. Infection is characterised by persistent viraemia and the presence of replicating virus in a wide range of anotomical sites, including bone marrow, lymphoid tissue, lung and liver [6-9]. As an indication of its great replicative capacity in vivo, studies of the kinetics of clearance of viraemia during interferon- $\alpha$ therapy have suggested that $>10^{10}$ TTV virions are produced every day, with $90 \%$ of the virus in plasma cleared and replenished over this period [10].

Even more remarkable is its genetic diversity. Broadly, TTV can be classified into four main genetic groups that show at least $50 \%$ nucleotide sequence divergence from each other. Each of the groups contains numerous genotypes differing by $c$. $30 \%$; to date, 28 distinct genotypes have been described [7,11,12], although this is undoubtedly an underestimate of the likely final number. At any one time, multiple genotypes may be found within an infected individual, often with different genotype combinations predominating in different 
tissues [7, 13]. Recently, a group of even more divergent viruses was discovered $[14,15]$; these viruses show a similar genome organisation to TTV but they are quite different in nucleotide sequence and genome length; the genome of these newly discovered viruses (described as TTV-like minivirus; TLMV) is almost 1000 bases shorter $(2.9 \mathrm{~kb})$. Infection with TLMV may also be ubiquitous in man and additional to infection with TTV. TLMV itself comprises a comparable number of diverse genetic groups and genotypes. At this stage, it is difficult to speculate on how many other small DNA viruses may be replicating in man.

Despite the great sequence diversity of TTV and TLMV, there is a highly conserved region in the virus's untranslated region (UTR) that has enabled the development of much more reliable PCR-based methods for detection of TTV, TLMV and TTV-related viruses in primates (Fig. 1) [16]. From such studies, close to ubiquitous infection with a range of increasingly genetically distinct TTV/TLMV-related viruses has been found in apes, Old World primate species, New World primates, and recently in the tree shrew [17], phylogenetically the most divergent member of the primate order. From the limited genetic characterisation of primate TTV-related viruses that has been achieved to date, it appears that their sequence relationships recapitulates that of their hosts, consistent with a process of long-term co-evoution. For example, chimpanzees harbour a range of genotypes of TTV and TLMV similar to and frequently interspersed with human genotypes; the existence of homologues of both TTV and TMLV in chimpanzees indicates their evo- lutionary lineages separated considerably earlier than the 5 million years estimated for host speciation. Old World and New World primates harbour viruses even more divergent than TTV and TLMV, with distinct genome sizes and very little sequence similarity in coding regions [16]. At the furthest extreme, the TTVlike virus detected in the tree shrew shares little but a common genome organisation with viruses from other primates [17].

Published information on the distribution of TTV and related viruses in other mammalian species may be misleading. Although it has been reported that TTV variants, similar to that of the prototype TTV strain, were detectable in a wide variety of farm animals and chickens [18], we have failed to find any evidence of TTV viraemia in a large survey of sheep, cows, goats and chickens, in tests with either the primers used in the original study or the more conserved UTR primers. Indeed, from the information gained from studies of primate viruses, it is more likely that if other mammals were infected with TTV-like viruses, they would be even more divergent in sequence from human TTV than the variants found in the tree shrew, i.e., so divergent that they would be undetectable even with the highly conserved primers from the UTR. Clearly, other methods for their detection may have to be developed to explore the distribution of TTV-related viruses in other mammals.

The only other known virus related to TTV is CAV. This virus shares some aspects of its genome organisation with TTV [19], such as the arginine-lysine rich

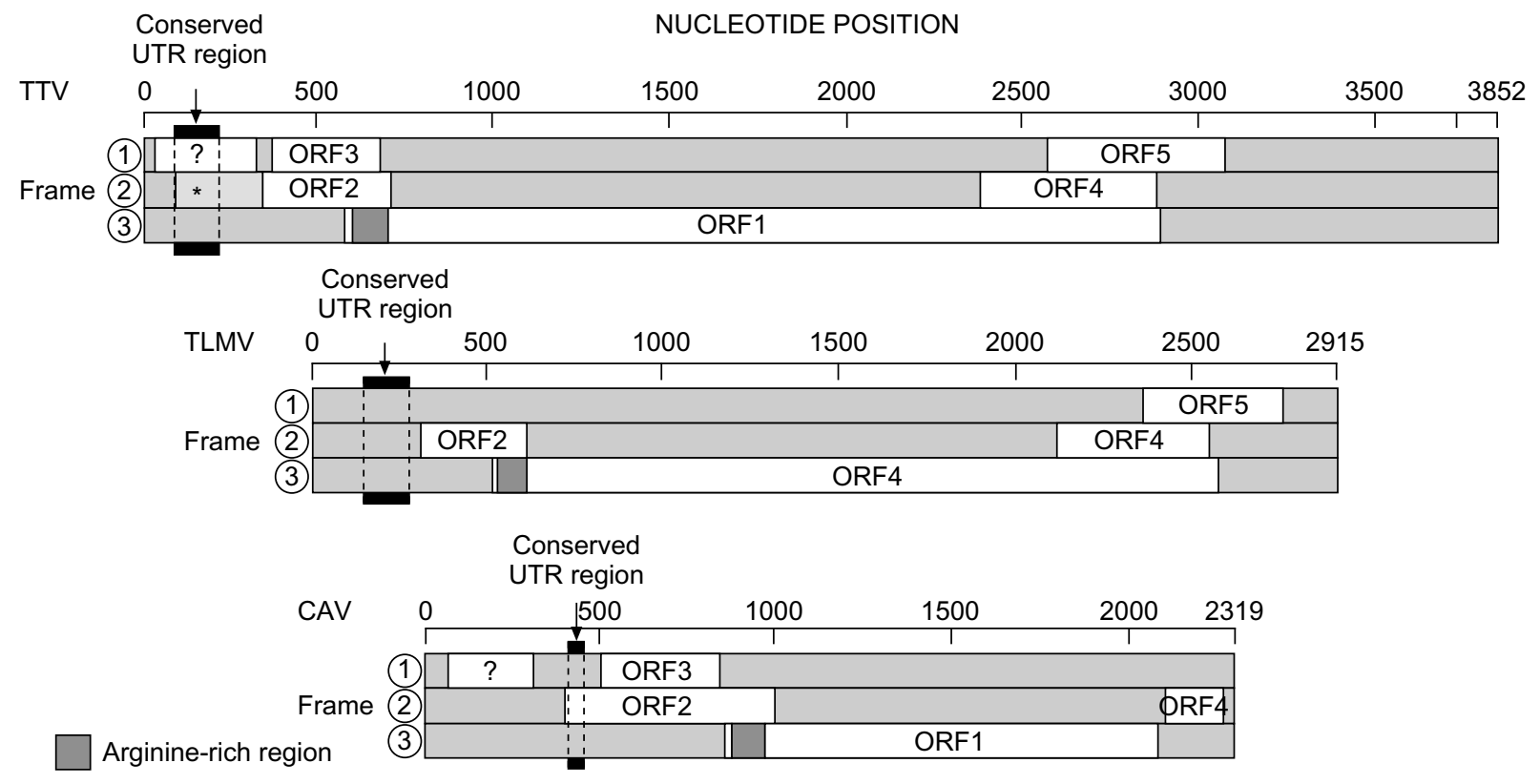

Fig. 1. Comparison of the genomes of TTV, TLMV and the distantly related virus CAV, shown in antisense, linearised configuration. Potential coding sequences shown as unfilled boxes (ORF1-ORF5), numbered as in [4] for TTV and TLMV, and in [19] for CAV. All three viruses are characterised by the partially overlapping reading frames ORF1 and ORF2, the former sharing the property of encoding highly basic amino acid residues at the amino terminus, indicated by $\square$. The position of the highly conserved region in the UTR of TTV, TLMV and CAV used for PCR is shown as a filled blocks above and below sequences. 
sequences at the amino terminus of ORF1, the overlap between ORF1 and 2 and, potentially, some aspects of gene transcription and splicing to generate mRNAs that express proteins from the three identified open reading frames (Fig. 1). From a co-evolution perspective, it might be supposed that CAV represents a virus split from the TTV/TLMV primate lineage even earlier in vertebrate evolution ( $>300$ million years ago). Therefore, this hypothesis implies that not only mammals but also all other land vertebrates, such as marsupials, reptiles, and possibly amphibians, could harbour TTVlike viruses.

\section{Disease associations of TTV}

The discovery that infection with TTV and TLMV is ubiquitous in man has rendered meaningless most of the earlier studies relating frequency of (detected) viraemia with acute or chronic hepatitis or other disease outcomes. Clearly, a virus that is present in all individuals cannot be primarily pathogenic per se. On the other hand, it is still contended by some groups that certain genotypes of TTV or TLMV may be specifically associated with disease. For example, following the suggestion from the original discoverers of TTV [20], it has been proposed that infection with the prototype genotype 1 strain of TTV may be associated with post-transfusion hepatitis [21]. More recently, infection with the TTV genotypes described as SENV$\mathrm{D}$ and SENV-H was found to be much more prevalent in patients with post-transfusion non-A-E hepatitis than transfused patients without hepatitis $(92 \%$ compared with 24\%) [22]. However, as is typical in TTV research, further work has tended to discount such an association $[23,24]$.

Part of the reason why previous investigations of the disease associations of TTV may have been so uninformative is that they have generally been based on the false premise that TTV is primarily a hepatotropic virus. More recent studies have demonstrated virus replication in a wide range of tissues, with a growing consensus that the bone marrow, lung and lymphoid tissue contain the highest levels of replicating virus [6-9]. The importance of the bone marrow as the source of replicating virus is perhaps illustrated by the observation of a disappearance of TTV viraemia in the myelosuppressed period immediately after bone marrow transplantation [25]. This cellular tropism may reflect the dependence of TTV on cell division for genome replication, a limitation shared with other small DNA viruses such as human parvovirus B19.

Recent studies have suggested that TTV replication is controlled by the activity of the immune system, as much higher circulating plasma virus loads are observed in immunodeficient individuals, such as those with AIDS [26,27] and with other intercurrent illnesses [8]. This situation closely resembles the rep- lication of the distantly related CAV in chickens. Anaemia, thrombocytopaenia and loss of $\mathrm{T}$ lymphocytes resulting from CAV replication in bone marrow and thymus are exacerbated by co-infection with other viruses, such as the immunosuppressive infections bursal disease virus [28]. Therefore, it is possible that TTV may have a similar adverse effect on bone marrow function, particularly in association with immune suppression. Indeed, some reports link TTV replication to thrombocytopenia and aplastic anaemia [29-31]. Of great potential interest is the possibility that many of the haematological complications of AIDS, such as neutropenia, thrombocytopenia and anaemia may be the direct result of increased TTV replication in the bone marrow. Supporting this hypothesis, our preliminary investigations have found exceptionally high virus loads in bone marrow of some individuals in terminal AIDS; in two cases, $>1000$ copies of TTV per bone marrow cell were detected, clearly a potentially harmful level of replication.

\section{Unique aspects of TTV}

In terms of their ubiquity and persistence, TTV and TLMV are comparable to the distributions of herpesviruses and polyomaviruses, but differ uniquely in their high replicative capacity that leads to life-long persistent viraemia in every human being. Even among the genotypes that infect man, their genetic diversity exceeds that of any other known virus, even that of the papillomaviruses infecting the skin. While the immune system appears to play a role in controlling virus replication [26,27], the mechanisms by which TTV establishes persistent infection remain unclear. While pre- or peri-natal infection may induce partial immunotolerance, that would not explain how infection with antigenically distinct genotypes of TTV can occur later in life [20,22].

As TTV and its relatives appear to have infected primates and probably all mammals throughout their evolutionary histories, an intriguing question is whether an absence of TTV infection would have any consequences, such as immune system dysregulation that may result from the absence of the substantial antigenic burden of TTV and TLMV in infected individuals. For all we know at present, TTV-related viruses may have not simply progressed from being parasitic to commensal, but may indeed now exist in a symbiotic relationship with their hosts. These unresolved questions undoubtedly represent fertile areas for fundamental virology and immunology research in the future.

PETER SIMMONDS,

Virus Evolution Group, Laboratory for Clinical and Molecular Virology, University of Edinburgh, Summerhall, Edinburgh, EH9 1QH, UK. 


\section{References}

1. McGeoch DJ, Cook S, Dolan A, Jamieson FE, Telford EAR Molecular phylogeny and evolutionary timescale for the family of mammalian herpesviruses. $J$ Mol Biol 1995; 247: 443-458.

2. Weigler BJ. Biology of B virus in macaque and human hosts: a review. Clin Infect Dis 1992; 14: 555-567.

3. Hahn BH, Shaw GM, De Cock KM, Sharp PM. AIDS as a zoonosis: scientific and public health implications. Science 2000; 287: 607-614.

4. Kamahora T, Hino S, Miyata $H$. Three spliced mRNAs of TT virus transcribed from a plasmid containing the entire genome in COS1 cells. $J$ Virol 2000; 74: 9980-9986.

5. Okamoto H, Mayumi M. TT virus: virological and genomic characteristics and disease associations. $J$ Gastroenterol 2001; 36: $519-529$.

6. Okamoto $\mathrm{H}$, Takahashi M, Nishizawa $\mathrm{T}$ et al. Replicative forms of TT virus DNA in bone marrow cells. Biochem Biophys Res Commun 2000; 270: 657-662.

7. Okamoto H, Nishizawa T, Takahashi M, Asabe S, Tsuda F, Yoshikawa A. Heterogeneous distribution of TT virus of distinct genotypes in multiple tissues from infected humans. Virology 2001; 228: 358-368.

8. Zhong S, Yeo W, Tang $\mathrm{M}$ et al. Frequent detection of the replicative form of TT virus DNA in a peripheral blood mononuclear cells and bone marrow cells in cancer patients. $J$ Med Virol 2002; 66: 428-434

9. Takahashi M, Asabe S, Gotanda Y, Kishimoto J, Tsuda F, Okamoto H. TT virus is distributed in various leukocyte subpopulations at distinct levels, with the highest viral load in granulocytes. Biochem Biophys Res Commun 2002; 290: $242-248$

10. Maggi F, Pistello M, Vatteroni $M$ et al. Dynamics of persistent TT virus infection, as determined in patients treated with alpha interferon for concomitant hepatitis $\mathrm{C}$ virus infection. $J$ Virol 2001; 75: 11999-12004.

11. Heller F, Zachoval R, Koelzer A, Nitschko H, Froesner GG. Isolate KAV: a new genotype of the TT-virus family. Biochem Biophys Res Commun 2001; 289: 937-941.

12. Hijikata M, Takahashi K, Mishiro S. Complete circular DNA genome of a TT virus variant (isolate name SANBAN) and 44 partial ORF2 sequences implicating a great degree of diversity beyond genotypes. Virology 1999; 260: 17-22.

13. Ball JK, Curran R, Berridge $\mathrm{S}$ et al. TT virus sequence heterogeneity in vivo: evidence for co-infection with multiple genetic types. J Gen Virol 1999; 80: 1759-1768.

14. Takahashi K, Iwasa Y, Hijikata M, Mishiro S. Identification of a new human DNA virus (TTV-like mini virus, TLMV) intermediately related to TT virus and chicken anemia virus. Arch Virol 2000; 145: 979-993.

15. Biagini $\mathrm{P}$, Gallian $\mathrm{P}$, Attoui $\mathrm{H}$ et al. Genetic analysis of fulllength genomes and subgenomic sequences of TT virus-like mini virus human isolates. J Gen Virol 2001; 82: 379-383.

16. Okamoto H, Nishizawa T, Tawara A et al. Species-specific TT viruses in humans and nonhuman primates and their phylogenetic relatedness. Virology 2000; 277: 368-378.

17. Okamoto H, Nishizawa T, Takahashi $\mathrm{M}$ et al. Genomic and evolutionary characterization of TT virus (TTV) in tupaias and comparison with species-specific TTVs in humans and nonhuman primates. $J$ Gen Virol 2001; 82: 2041-2050.

18. Leary, TP, Erker JC, Chalmers ML, Desai SM, Mushahwar IK. Improved detection systems for TT virus reveal high prevalence in humans, non-human primates and farm animals. J Gen Virol 1999; 80: 2115-2120.

19. Kato A, Fujino M, Nakamura T, Ishihama A, Otaki Y. Gene organization of chicken anemia virus. Virology 1995; 209: 480-488.

20. Nishizawa T, Okamoto H, Konishi K, Yoshizawa H, Miyakawa Y, Mayumi M. A novel DNA virus (TTV) associated with elevated transaminase levels in posttransfusion hepatitis of unknown etiology. Biochem Biophys Res Commun 1997; 241 92-97.

21. Tanaka Y, Hayashi J, Ariyama I, Furusyo N, Etoh Y, Kashiwagi S. Seroepidemiology of TT virus infection and relationship between genotype and liver damage. Dig Dis $\mathrm{Sc}$ 2000; 45: 2214-2220.

22. Umemura T, Yeo AE, Sottini A et al. SEN virus infection and its relationship to transfusion-associated hepatitis. Hepatology 2001; 33: 1303-1311.

23. Debiaggi M, Zara F, Sacchi $\mathrm{P}$ et al. Transfusion-transmitted virus infection in HIV-1-seropositive patients. Clin Microbiol Infect 2000; 6 246-250.

24. Shibata M, Wang RYH, Yoshiba M, Shih JW-K, Alter HJ, Mitamura K. The presence of a newly identified infectious agent (SEN virus) in patients with liver diseases and in blood donors in Japan. J Infect Dis 2001; 184: 400-404.

25. Kanda Y, Tanaka Y, Kami M et al. TT virus in bone marrow transplant recipients. Blood 1999; 93: 2485-2490.

26. Touinssi $\mathrm{M}$, Gallian $\mathrm{P}$, Biagini $\mathrm{P}$ et al. TT virus infection: prevalence of elevated viraemia and arguments for the immune control of viral load. J Clin Virol 2001; 21: 135-141.

27. Shibayama T, Masuda G, Ajisawa A et al. Inverse relationship between the titre of TT virus DNA and the CD4 cell count in patients infected with HIV. AIDS 2001; 15: 563-570.

28. Imai K, Mase M, Tsukamoto K, Hihara H, Yuasa N. Persisten infection with chicken anaemia virus and some effects of highly virulent infectious bursal disease virus infection on its persistency. Res Vet Sci 1999; 67: 233-238.

29. Tokita H, Murai S, Kamitsukasa $\mathrm{H}$ et al. Influence of TT virus infection on the thrombocytopenia of patients with chronic liver disease. Hepatol Res 2001; 20: 288-300

30. Miyamoto M, Takahashi H, Sakata I, Adachi Y. Hepatitisassociated aplastic anemia and transfusion-transmitted virus infection. Intern Med 2000; 39: 1068-1070.

31. Safadi R, Or R, Ilan Y et al. Lack of known hepatitis virus in hepatitis-associated aplastic anemia and outcome after bone marrow transplantation. Bone Marrow Transplant 2001; 27: $183-190$. 